\title{
Role of adiponectin in the relationship between visceral adiposity and fibroblast growth factor 23 in non-diabetic men with normal kidney function
}

\author{
Yuka Natsuki ${ }^{1)}$, Tomoaki Morioka ${ }^{1}$, Shinya Fukumoto ${ }^{2}$, Yoshinori Kakutani ${ }^{1)}$, Yuko Yamazaki ${ }^{1)}$, \\ Akinobu Ochi ${ }^{1)}$, Masafumi Kurajoh ${ }^{1)}$, Katsuhito Mori ${ }^{3)}$, Tetsuo Shoji ${ }^{4)}$, Yasuo Imanishi ${ }^{1)}$, Masaaki Inaba ${ }^{1)}$ \\ and Masanori Emoto ${ }^{1)}$
1) Department of Metabolism, Endocrinology and Molecular Medicine, Osaka City University Graduate School of Medicine, Osaka 545-8585, Japan
2) Department of Premier Preventive Medicine, Osaka City University Graduate School of Medicine, Osaka 545-8585, Japan
3) Department of Nephrology, Osaka City University Graduate School of Medicine, Osaka 545-8585, Japan \\ 4) Department of Vascular Medicine, Osaka City University Graduate School of Medicine, Osaka 545-8585, Japan
}

\begin{abstract}
Fibroblast growth factor 23 (FGF23) is a key regulator of phosphate metabolism. Circulating FGF23 levels are associated with obesity, metabolic syndrome, and cardiovascular disease in the general population, but the underlying mechanism remains unclear. Therefore, we aimed to determine the associations between serum FGF23 levels and visceral adiposity as well as serum adiponectin levels in 189 adults without diabetes and with normal kidney function who were selected from the MedCity 21 health examination registry. The exclusion criteria included diabetes mellitus or impaired kidney function (estimated glomerular filtration rate [eGFR] $<60 \mathrm{~mL} / \mathrm{min} / 1.73 \mathrm{~m}^{2}$ ). Levels of serum FGF23 and total adiponectin, and visceral fat area (VFA) on computed tomography images were measured. Serum FGF23 levels were higher and VFA was greater, whereas serum adiponectin levels were lower in men than in women. Serum FGF23 levels positively correlated with VFA in men; they remained marginally significant after adjusting for age, eGFR, and serum levels of calcium, phosphate, intact parathyroid hormone, and 1,25-dihydroxyvitamin D. Importantly, when serum adiponectin levels were included as a covariate, serum adiponectin levels comprised an independent determinant of serum FGF23 levels in men, whereas VFA did not. In conclusion, lower serum adiponectin, rather than a greater VFA, was associated with higher serum FGF23 levels in non-diabetic men with normal kidney function. These findings suggest that adiponectin plays a role in the relationship between visceral adiposity and FGF23 in men.
\end{abstract}

Key words: Fibroblast growth factor 23, Adiponectin, Visceral fat, Obesity

DYSREGULATED BONE and mineral metabolism is an important feature of obesity, diabetes, and cardiovascular disease (CVD), even in the absence of chronic kidney disease (CKD) [1-3]. Several humoral factors derived from accumulated adipose tissue, including adipocytokines, inflammatory cytokines, and fatty acids, adversely affect bone and mineral homeostasis $[4,5]$.

Fibroblast growth factor 23 (FGF23), produced and secreted by osteocytes in bone, regulates phosphate and vitamin D metabolism [6-8]. Increased circulating

Submitted Mar. 16, 2021; Accepted Aug. 10, 2021 as EJ21-0185 Released online in J-STAGE as advance publication Sep. 8, 2021 Correspondence to: Tomoaki Morioka, M.D, Ph.D., Department of Metabolism, Endocrinology and Molecular Medicine, Osaka City University Graduate School of Medicine, 1-4-3, Asahi-machi, Abeno-ku, Osaka 545-8585 Japan.

E-mail: m-tomo@med.osaka-cu.ac.jp
FGF23 levels in conjunction with reduced kidney function in CKD [6], are independently associated with risk of CVD events and mortality [9, 10]. Increased FGF23 levels are also associated with the risk of CVD events and mortality in individuals without CKD or in general populations [11-13].

Recent studies have shown that elevated FGF23 levels are associated with greater adiposity [14-21], metabolic syndrome [19], and cardiovascular risk factors [16, 17, 19] in general populations. Notably, associations between FGF23 levels and adiposity [18-21] as well as metabolic risk factors [17, 19] are independent of potential confounders including age, estimated glomerular filtration rate (eGFR), calcium, and phosphate levels. These findings suggest a close relationship between FGF23 and adiposity and obesity-related metabolic risk factors; however, the underlying mechanism remains unclear. 
Adiponectin is an anti-inflammatory adipocytokine that is exclusively synthesized by adipocytes and exerts beneficial effects on metabolic diseases and CVDs [22, 23]. Adiponectin directly acts on osteoblasts and osteoclasts to regulate bone mineral density and bone turnover markers, suggesting that it functions in the endocrine loop between bone and adipose tissue [24, 25]. Moreover, adiponectin regulates the calcium and phosphate balance and renal mineral excretion through FGF23/ Klotho, implying that interaction between adiponectin and FGF23 regulates bone metabolism [26].

The mechanism underlying the association between FGF23 and obesity as well as risk of CVD has not yet been clinically investigated. Therefore, we aimed to determine relationships between serum FGF23 levels and visceral adiposity, and the involvement of adiponectin in the association between FGF23 and obesity. Similar to previous studies $[9,14,16,18-21]$, we assayed intact FGF23, but not C-terminal FGF23, because the assay of latter detects a biologically inactive $\mathrm{C}$-terminal fragment that is affected by metabolic changes caused by visceral obesity $[6,8]$. Because visceral adiposity generally differs between men and women, and because levels of FGF23 [27, 28] and adiponectin [23, 29, 30] are significantly affected by diabetes and CKD, we separately analyzed data from adult men and women without diabetes or kidney dysfunction.

\section{Materials and Methods}

\section{Participants and study design}

This single-center, cross-sectional study initially selected 251 consecutive individuals from the MedCity21 Health Examination Registry who underwent comprehensive medical examinations at MedCity21, Osaka City University Hospital Advanced Medical Center for Preventive Medicine between June 2015 and November 2017. Sixty-two of the initially selected 251 individuals were excluded based on the following criteria: diabetes mellitus $(n=27)$, defined according to the criteria of the American Diabetes Association [31] and the Japan Diabetes Society [32], current or previous medication with anti-diabetic agents, eGFR $<60 \mathrm{~mL} / \mathrm{min} / 1.73 \mathrm{~m}^{2}$ $(n=22)$, and missing data about mineral metabolites and adiposity $(n=13)$. Finally, 189 participants were enrolled. Current or ex-smokers were defined as smokers.

This study proceeded in accordance with the Declaration of Helsinki (2013 amendment) and the Ethical Guidelines for Medical Research Involving Human Subjects (Japanese Ministry of Health, Labour and Welfare, 2014). We collected data from the biorepository of the MedCity21 Health Examination Registry, which was approved by the Ethics Committee of the Osaka City
University Graduate School of Medicine (Approval no: 2927). The participants provided consent to a broad range of uses of their data and samples to develop advanced diagnostic techniques to treat and prevent cancer, diabetes, mental disorders, dyslipidemia, hypertension, hyperuricemia, obesity, cardiovascular, cerebrovascular, chronic respiratory, liver, digestive, gynecological, and skin diseases. The Ethics Committee of the Osaka City University Graduate School of Medicine (Approval no: 4417) approved the study, and informed consent was obtained using the opt-out method on the MedCity21 website (http://www.medcity21.jp/ index.shtml).

\section{Physical and laboratory measurements}

Information about smoking habits, present and past illnesses, and medications was obtained. Blood pressure was conventionally measured in the participants after at least 15 min of rest, using a cuff with an automated sphygmomanometer. Blood was drawn after an overnight fast and biochemical parameters were measured using standard laboratory MedCity21 protocols at the Central Laboratory, and the remaining blood samples were stored at $-80^{\circ} \mathrm{C}[33,34]$. The GFR was estimated using the Japanese eGFR equation [35]. Glycated hemoglobin A1c (HbA1c) was assessed as the National Glycohemoglobin Standardization Program equivalent value (NGSP, \%) according to the guidelines of the Japan Diabetes Society [32]. Serum immunoreactive insulin levels were measured using electrochemiluminescence immunoassays (Roche Holdings AG, Basel, Switzerland). The homeostasis model assessment of insulin resistance (HOMA-R) was calculated as: fasting insulin $(\mu \mathrm{U} / \mathrm{mL}) \times$ fasting glucose $(\mathrm{mg} / \mathrm{dL}) / 405$ [29, 33, 34, 36].

Serum levels of human intact FGF23 were measured using sandwich enzyme-linked immunosorbent assay kits (Kainos Laboratories, Tokyo, Japan) [28, 37]. Fasting serum adiponectin levels were measured using latex particle-enhanced turbidimetric immunoassays (Otsuka Pharmaceutical Co., Tokyo, Japan) [33, 34]. Intact parathyroid hormone $(\mathrm{PTH})$ and 1,25-dihydroxyvitamin D $\left(1,25(\mathrm{OH})_{2} \mathrm{D}\right)$ levels were measured in frozen serum samples using immunoradiometric assays and radioimmunoassays, respectively, at SRL, Inc. (Tokyo, Japan) [28].

\section{Measurement of visceral fat area}

Visceral fat area (VFA) was measured using computed tomography (CT) as described previously [33, 34]. Briefly, VFA values were calculated using fat-pointer software version 2 (Hitachi, Ltd., Tokyo, Japan), from a single 5-mm slice at the level of umbilicus on CT images acquired using the Supria Grande system (Hitachi, Ltd.). 


\section{Statistics}

Data are expressed as numbers (\%) or medians with interquartile ranges. Data from men and women were compared using $\chi^{2}$-tests or Wilcoxon rank-sum tests. Correlations between serum FGF23 levels and anthropometric or metabolic parameters were assessed using Spearman's rank correlation tests. Independent determinants of FGF23 levels after adjusting for potential confounders including age, VFA, eGFR, and serum calcium, phosphate, intact $\mathrm{PTH}, 1,25(\mathrm{OH})_{2} \mathrm{D}$, and adiponectin levels were determined using multiple regression analysis. A two-sided $p$-value of $<0.05$ was considered significant. All data were statistically analyzed using JMP 14 (SAS Institute Inc., Cary, NC, USA).

\section{Results}

\section{Clinical characteristics, serum FGF23 and adiponectin levels, and visceral adiposity in the participants}

Table 1 shows the characteristics of the study participants. The median age was 52 years and the median body mass index (BMI) was $22.4 \mathrm{~kg} / \mathrm{m}^{2}$. By definition, kidney function and glucose metabolism were within the normal range in all participants. The BMI, diastolic blood pressure, uric acid, fasting glucose, immunoreactive insulin, HOMA-R, and triglycerides were higher, while high-density lipoprotein cholesterol was lower in men than in women. The prevalence of smoking and of medication with antihypertensive agents was higher in men than in women.

The median values for phosphate, FGF23, intact PTH, $1,25(\mathrm{OH})_{2} \mathrm{D}$, and total adiponectin in serum, and VFA in the total population were $3.3 \mathrm{mg} / \mathrm{dL}, 29.7 \mathrm{pg} / \mathrm{mL}, 37.0$ $\mathrm{pg} / \mathrm{mL}, 63.4 \mathrm{pg} / \mathrm{mL}, 8.8 \mu \mathrm{g} / \mathrm{mL}$, and $65.1 \mathrm{~cm}^{2}$, respectively. Men had lower serum phosphate and adiponectin, as well as higher serum FGF23 and $1,25(\mathrm{OH})_{2} \mathrm{D}$ levels and greater VFA than women. The eGFR did not significantly differ between men and women (Table 1).

\section{Correlations between serum FGF23 levels and anthropometric and metabolic parameters}

We assessed unadjusted correlations between serum FGF23 levels and anthropometric or metabolic parameters (Table 2). Serum FGF23 levels positively correlated with age, BMI, VFA, serum calcium, and uric acid levels, and negatively with eGFR and serum adiponectin levels in the total population. Levels of phosphate, intact $\mathrm{PTH}$, and $1,25(\mathrm{OH})_{2} \mathrm{D}$ in serum did not significantly correlate with those of FGF23 in the total population. Serum FGF23 levels correlated with greater VFA, older age, and higher uric acid levels, but not with BMI, in men (Fig. 1a and Table 2). On the contrary, serum FGF23 levels significantly correlated with eGFR, serum calcium, phosphate, and intact PTH, but not with VFA or BMI in women (Fig. 1b and Table 2).

\section{Multivariate analysis of determinants of serum FGF23 levels}

We assessed whether the association between FGF23 and visceral adiposity was independent of potential confounders in men and women using multiple regression analysis (Table 3). The association between serum FGF23 levels and VFA in men remained marginally significant $(\beta=0.173, p=0.097)$ after adjustment for age, eGFR, and serum calcium, phosphate, intact PTH, and $1,25(\mathrm{OH})_{2} \mathrm{D}$ levels (Model 1). Serum levels of adiponectin and FGF23 were also independently associated (Model 2). Furthermore, serum adiponectin was an independent determinant of serum FGF23 levels in men ( $\beta=$ $-0.251, p=0.039$ ), whereas VFA was not (Model 3). Serum levels of calcium and FGF23 were independently associated in women (all models). VFA was associated with FGF23 levels in women $(\beta=0.256, p=0.021)$ as in men (Model 1), but serum levels of adiponectin and FGF23 were not significantly associated in women (Models 2 and 3).

In agreement with the results of the univariate analyses, multivariate analysis found no significant association between BMI and serum FGF23 levels after adjusting for age, eGFR, and bone mineral metabolism markers in men $(\beta=0.135, p=0.182)$ or women $(\beta=$ $0.104, p=0.282)$.

\section{Discussion}

This study investigated the relationship between serum FGF23 levels and visceral adiposity and the possible involvement of adiponectin in the relationship between FGF23 and obesity in non-diabetic adult men and women with normal kidney function. Our results confirmed that serum FGF23 levels positively correlated with VFA in men, and that the association remained marginally significant after adjusting for confounders, including kidney function and bone mineral metabolism markers. Importantly, the association between serum FGF23 levels and VFA was no longer significant after further adjustment for serum adiponectin levels. To the best of our knowledge, this is the first study to suggest that adiponectin plays a role in the relationship between serum FGF23 levels and visceral adiposity in men.

A simple correlation analysis associated serum FGF23 levels with visceral adiposity, rather than BMI. This result was largely in line with previous findings of an association between serum FGF23 levels and abdominal adiposity in a general population determined using $\mathrm{CT}$ 
Table 1 Clinical characteristics, intact FGF23 levels, adiponectin levels, and visceral adiposity in all participants and in men and women separately

\begin{tabular}{|c|c|c|c|c|}
\hline & Total & Men & Women & $p$ \\
\hline$N$ & 189 & 94 & 95 & - \\
\hline Age (years) & $52[45-63]$ & $52[44-63]$ & $53[45-63]$ & 0.411 \\
\hline BMI $\left(\mathrm{kg} / \mathrm{m}^{2}\right)$ & $22.4[20.8-24.8]$ & $23.9[21.7-25.2]$ & $21.4[19.6-23.1]$ & $<0.001$ \\
\hline Systolic blood pressure (mmHg) & $119[109-131]$ & $123[112-131]$ & $117[106-131]$ & 0.324 \\
\hline Diastolic blood pressure (mmHg) & $74[66-82]$ & 77 [71-83] & $70[65-80]$ & $<0.001$ \\
\hline Smoker, $n(\%)$ & $105(55.6)$ & $72(76.6)$ & $33(34.7)$ & $<0.001$ \\
\hline Antihypertensive drugs, $n(\%)$ & $29(15.3)$ & $21(22.3)$ & $8(8.4)$ & 0.032 \\
\hline Antihyperlipidemic drugs, $n(\%)$ & $21(11.1)$ & $12(12.8)$ & $9(9.5)$ & 0.988 \\
\hline Creatinine (mg/dL) & $0.70[0.61-0.82]$ & $0.81[0.75-0.90]$ & $0.61[0.55-0.65]$ & $<0.001$ \\
\hline eGFR (mL/min/1.73 m²) & $77.8[70.3-88.6]$ & $77.3[71.2-87.9]$ & $81.0[69.6-90.0]$ & 0.420 \\
\hline Serum calcium (mg/dL) & $9.4[9.2-9.6]$ & $9.4[9.2-9.6]$ & $9.4[9.1-9.6]$ & 0.199 \\
\hline Serum phosphate (mg/dL) & $3.3[3.0-3.7]$ & $3.1[2.9-3.4]$ & $3.5[3.2-3.8]$ & $<0.001$ \\
\hline Uric acid $(\mathrm{mg} / \mathrm{dL})$ & $5.4[4.3-6.4]$ & $6.1[5.6-7.0]$ & $4.4[3.8-5.3]$ & $<0.001$ \\
\hline Fasting glucose (mg/dL) & 99 [94-105] & $102[96-107]$ & $96[92-102]$ & $<0.001$ \\
\hline HbAlc (\%) & $5.6[5.4-5.8]$ & $5.6[5.4-5.8]$ & $5.6[5.5-5.9]$ & 0.324 \\
\hline Immunoreactive insulin $(\mu \mathrm{U} / \mathrm{mL})$ & $5.5[3.8-8.0]$ & $5.9[4.1-8.5]$ & $4.8[3.5-7.1]$ & 0.017 \\
\hline HOMA-R & $1.3[0.9-2.0]$ & $1.5[1.0-2.2]$ & $1.2[0.8-1.7]$ & 0.005 \\
\hline Triglycerides (mg/dL) & $86[63-123]$ & $101[77-134]$ & $73[54-96]$ & $<0.001$ \\
\hline Total cholesterol (mg/dL) & 204 [181-222] & $197[178-218]$ & $210[185-230]$ & 0.023 \\
\hline HDL-cholesterol (mg/dL) & $60[50-71]$ & $52[42-64]$ & $65[57-77]$ & $<0.001$ \\
\hline LDL-cholesterol (mg/dL) & 116 [96-136] & 115 [99-136] & $120[93-134]$ & 0.914 \\
\hline Intact FGF23 (pg/mL) & $29.7[18.5-45.4]$ & $36.7[23.9-51.4]$ & $26.4[15.2-38.4]$ & 0.003 \\
\hline Intact PTH (pg/mL) & $37.0[31.0-46.0]$ & $37.0[31.0-47.3]$ & $36.0[31.0-46.0]$ & 0.369 \\
\hline $1,25(\mathrm{OH})_{2} \mathrm{D}(\mathrm{pg} / \mathrm{mL})$ & $63.4[51.2-74.0]$ & $67.9[56.0-78.4]$ & $58.2[47.8-69.9]$ & $<0.001$ \\
\hline Adiponectin $(\mu \mathrm{g} / \mathrm{mL})$ & $8.8[6.1-11.8]$ & $6.6[5.3-8.8]$ & $10.7[8.6-15.5]$ & $<0.001$ \\
\hline VFA $\left(\mathrm{cm}^{2}\right)$ & $65.1[37.3-100.4]$ & 88.6 [60.8-118.2] & $44.4[24.0-71.2]$ & $<0.001$ \\
\hline
\end{tabular}

Data are median [interquartile range] or $n(\%) . P$-values by Wilcoxon rank-sum test or $\chi^{2}$-test for comparison between men and women. FGF, fibroblast growth factor; BMI, body mass index; eGFR estimated glomerular filtration rate; HbA1c glycated hemoglobin A1c; HOMA-R, homeostasis model assessment of insulin resistance; HDL, high-density lipoprotein; LDL, low-density lipoprotein; PTH, parathyroid hormone; $1,25(\mathrm{OH})_{2} \mathrm{D}, 1,25$-dihydroxyvitamin $\mathrm{D}$; VFA, visceral fat area.

[21], magnetic resonance imaging [18, 20], or dual X-ray absorptiometry $[16,19]$. As in the present study, most of those findings $[18,19,21]$ indicated that abdominal adipose tissue mass was more effective than BMI as a determinant of serum FGF23 levels, and that it was independent of known confounders including eGFR and calcium/phosphate levels.

The mechanism underlying the relationship between FGF23 and abdominal adiposity in humans remains unclear. A causal role of FGF23 in the regulation of body adiposity has been suggested by findings from a study on Fgf23-deficient mice, which have a lower body fat mass than wild-type mice [38]. However, increased FGF23 concentrations might be a downstream consequence of obesity, because studies of weight loss therapy for obese adults have found a significant decline in serum FGF23 levels after sleeve gastrectomy [39] or calorie restriction [16]. Nevertheless, unknown factors other than body fat mass itself might regulate FGF23 levels because changes in FGF23 concentrations and in fat mass after weight loss did not significantly correlate in the study of sleeve gastrectomy [39].

The present study showed that VFA and serum adiponectin levels were independently associated with serum 
Table 2 Correlations between intact FGF23 levels and anthropometric and metabolic parameters

\begin{tabular}{|c|c|c|c|c|c|c|}
\hline & \multicolumn{2}{|c|}{ Total } & \multicolumn{2}{|c|}{ Men } & \multicolumn{2}{|c|}{ Women } \\
\hline & $\rho$ & $p$ & $\rho$ & $p$ & $\rho$ & $p$ \\
\hline Age & 0.163 & 0.025 & 0.288 & 0.005 & 0.079 & 0.446 \\
\hline BMI & 0.161 & 0.027 & 0.158 & 0.129 & 0.062 & 0.552 \\
\hline Systolic blood pressure & 0.079 & 0.281 & 0.164 & 0.117 & 0.006 & 0.955 \\
\hline eGFR & -0.228 & 0.002 & -0.198 & 0.056 & -0.235 & 0.022 \\
\hline Serum calcium & 0.234 & 0.001 & 0.132 & 0.206 & 0.282 & 0.006 \\
\hline Serum phosphate & 0.127 & 0.083 & 0.113 & 0.277 & 0.372 & $<0.001$ \\
\hline Uric acid & 0.253 & $<0.001$ & 0.241 & 0.019 & 0.106 & 0.306 \\
\hline $\mathrm{HbA} 1 \mathrm{c}$ & 0.069 & 0.344 & 0.177 & 0.088 & 0.007 & 0.950 \\
\hline HOMA-R & 0.114 & 0.120 & 0.162 & 0.119 & -0.006 & 0.956 \\
\hline Triglycerides & 0.097 & 0.185 & 0.050 & 0.636 & 0.018 & 0.865 \\
\hline HDL-cholesterol & -0.020 & 0.784 & 0.050 & 0.634 & 0.119 & 0.250 \\
\hline LDL-cholesterol & -0.015 & 0.838 & -0.066 & 0.530 & 0.021 & 0.838 \\
\hline Intact PTH & -0.126 & 0.083 & -0.040 & 0.724 & -0.240 & 0.019 \\
\hline $1,25(\mathrm{OH})_{2} \mathrm{D}$ & -0.013 & 0.857 & -0.114 & 0.274 & -0.052 & 0.619 \\
\hline Adiponectin & -0.156 & 0.032 & -0.121 & 0.243 & 0.037 & 0.724 \\
\hline VFA & 0.243 & $<0.001$ & 0.269 & 0.009 & 0.131 & 0.207 \\
\hline
\end{tabular}

$\rho$, Spearman's rank correlation coefficient. Abbreviations are as in Table 1.

FGF23, while serum FGF23 was independently associated in men with serum adiponectin rather than VFA when the model included both VFA and serum adiponectin as covariates. These results indicated that the relationship between VFA and FGF23 is mediated by adiponectin. Circulating adiponectin levels are reduced in obese humans, particularly in those with visceral obesity [22, 23]. Hypoadiponectinemia is also directly or indirectly associated with various pathological conditions, including insulin resistance, type 2 diabetes, metabolic syndrome, dyslipidemia, hypertension, oxidative stress, and CVDs [22, 23]. The association between serum adiponectin and serum FGF23 levels was independent of VFA and known confounders of FGF23 levels, such as eGFR and phosphate levels; thus, reduced adiponectin levels, rather than increased visceral adiposity, might be an independent factor that determines increased serum levels of FGF23, which is derived from osteocytes in bone. The inverse relationship between adiponectin and FGF23 can be explained as follows.

One explanation is that the amount of dietary intake is a key factor in the determination of FGF23 and adiponectin levels. Although the mechanism remains obscure, circulating FGF23 levels increase in response to dietary phosphate load to maintain normal serum phosphate levels, even in individuals with normal kidney function $[40,41]$. Given that dietary phosphate consumption closely correlates with total energy intake in the general population [42], high dietary phosphate intake would not only increase FGF23 levels but also reduce adiponectin levels via increased visceral adiposity. We did not have the dietary information about the participants, and were thus unable to clarify the impact of dietary factors on serum FGF23 and adiponectin levels.

Another explanation is that reduced adiponectin levels might be causally associated with increased serum FGF23 levels. Clinical studies have consistently shown that serum adiponectin levels are negatively and positively associated with bone mineral density [43-47] and markers of bone turnover, respectively [45], suggesting that adiponectin negatively regulates bone mass and bone metabolism [24, 25]. A direct effect of adiponectin on bone metabolism is supported by the expression of adiponectin receptors in human and murine osteoblasts [24, 25, 48, 49]. In addition, adiponectin directly reduces FGF23 production in cultured osteocytes in vitro, although the expression of adiponectin receptors in cultured osteocytes has not been analyzed [26]. Considering that FGF23 is produced by late-stage osteoblasts and early osteocytes [50], these findings could support a causal effect of adiponectin on FGF23 production in bone-forming cells.

The mechanistic basis for adiponectin in the regulation of mineral homeostasis was recently investigated in mice 
$\mathbf{a}$

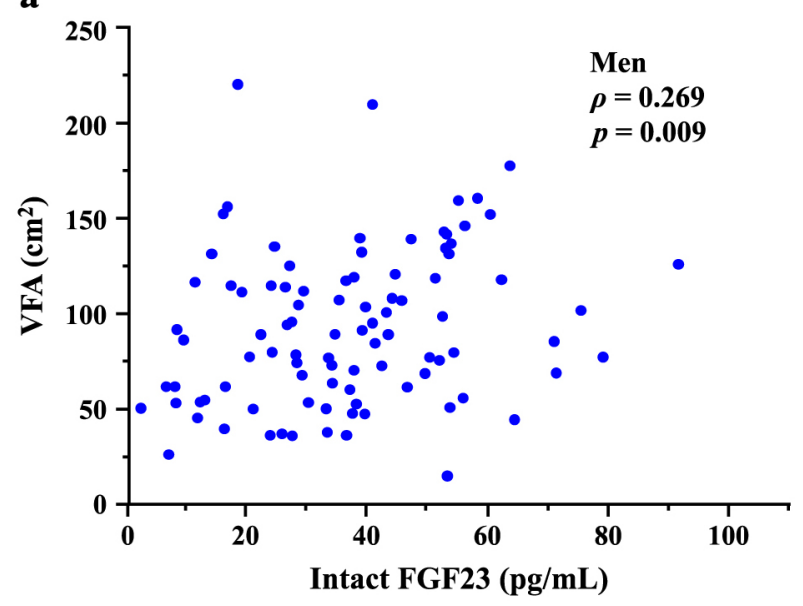

b

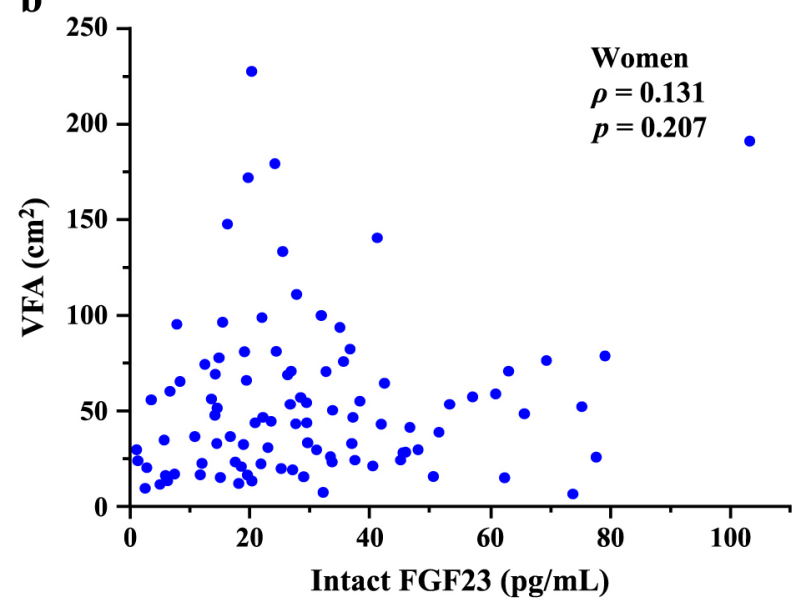

Fig. 1 Correlation between intact fibroblast growth factor (FGF23) levels and visceral fat area (VFA) in men (a) and in women (b)

$\rho$, Spearman's rank correlation coefficient.

with a wide range of adiponectin levels and in cultured cells [26]. Adiponectin was found to induce bone loss and renal calcium excretion and directly reduce Klotho release from renal tubular epithelial cells. In addition, lower serum levels of FGF23 were found in adiponectintransgenic mice than in wild-type mice on a normal chow diet. Moreover, adiponectin directly inhibited FGF23 production in cultured osteocytes, suggesting an inhibitory effect of adiponectin on FGF23 secretion. Consistent with these findings, serum FGF23 level inversely correlated with serum adiponectin levels after adjustment for age, sex, BMI, phosphate, albumin, calcium, vitamin D, PTH, and eGFR in a community-based cohort of elderly persons [19]. The association between serum adiponectin and FGF23 levels was adjusted for BMI in that study, but not for body fat mass, as in our study. Taken together, our findings provide additional evidence supporting an inverse relationship between adi- ponectin and FGF23 and we are the first to suggest that adiponectin mediates the effect of visceral adiposity on FGF23 levels in humans.

We identified an association between FGF23 levels and VFA in men $(\beta=0.173, p=0.097)$ and women $(\beta=$ $0.256, p=0.021)$, whereas the association between serum FGF23 and adiponectin levels differed between them (men, $\beta=-0.251, p=0.039$; women, $\beta=-0.098, p$ $=0.403$ ) (Table 3 ). These findings suggested a sexspecific association of FGF23 with adiponectin, but not VFA. Large-scale, population-based studies have shown a significant association between FGF23 levels and measures of adiposity, after adjustment for sex [15, 19, $20]$ and in men and women when analyzed separately $[18,19]$. Our results are consistent with these findings and suggest that the association between visceral adiposity and FGF23 levels is sex-independent.

To date, only one study has shown a link between adiponectin and FGF23 levels, which remained significant even after adjustment for sex differences in a community-based cohort of elderly persons [19]. The results of our study do not conflict with these findings, because an interaction analysis indicated that the association between adiponectin levels and FGF23 levels was not affected by sex differences ( $p$ for interaction, 0.373 ). A significant difference in VFA or adiponectin levels between women and men might have caused a sex difference in the association between FGF23 and adiponectin in our study. In addition, our results indicated that serum levels of calcium, phosphate, PTH, or vitamin D did not dominantly affect serum FGF23 levels. This is consistent with prior findings showing that parameters of obesity or lipid metabolism and those of bone mineral metabolism similarly impact on FGF23 levels in the general population with normal kidney function [16, 17].

This study has several limitations. Because this study included only middle-aged individuals without diabetes or kidney dysfunction, the results cannot be directly applied to other populations. Our conclusions might be overestimated or underestimated, as they were based on single measurements of VFA, serum FGF23, and serum adiponectin. We did not include serum leptin levels [51, 52], menopausal status, and hormone replacement therapy in women as adjustment factors for serum FGF23 levels. Lastly, because of its cross-sectional design, we could not elucidate the causal relationship between levels of FGF23 and adiponectin or visceral adiposity.

In conclusion, lower levels of serum adiponectin, rather than greater visceral adiposity, were associated with higher serum FGF23 levels in non-diabetic men with normal kidney function. Our results suggested that adiponectin plays a role in the relationship between visceral adiposity and FGF23. This study provides evidence 
Table 3 Multivariate analysis of the determinants of intact FGF23 levels

\begin{tabular}{|c|c|c|c|c|c|c|c|c|c|c|c|c|}
\hline & \multicolumn{6}{|c|}{ Men } & \multicolumn{6}{|c|}{ Women } \\
\hline & \multicolumn{2}{|c|}{ Model 1} & \multicolumn{2}{|c|}{ Model 2} & \multicolumn{2}{|c|}{ Model 3} & \multicolumn{2}{|c|}{ Model 1} & \multicolumn{2}{|c|}{ Model 2} & \multicolumn{2}{|c|}{ Model 3} \\
\hline & $\beta$ & $p$ & $\beta$ & $p$ & $\beta$ & $p$ & $\beta$ & $p$ & $\beta$ & $p$ & $\beta$ & $p$ \\
\hline Age & 0.227 & 0.045 & 0.333 & 0.003 & 0.320 & 0.008 & -0.112 & 0.329 & 0.015 & 0.895 & -0.076 & 0.533 \\
\hline eGFR & -0.095 & 0.375 & -0.113 & 0.281 & -0.108 & 0.306 & -0.182 & 0.100 & -0.190 & 0.097 & -0.199 & 0.078 \\
\hline Serum calcium & 0.234 & 0.024 & 0.240 & 0.018 & 0.242 & 0.018 & 0.324 & 0.002 & 0.343 & 0.001 & 0.324 & 0.002 \\
\hline Serum phosphate & 0.091 & 0.374 & 0.089 & 0.372 & 0.089 & 0.377 & 0.205 & 0.070 & 0.200 & 0.086 & 0.223 & 0.054 \\
\hline Intact PTH & 0.008 & 0.942 & -0.011 & 0.910 & -0.010 & 0.925 & -0.018 & 0.861 & -0.004 & 0.971 & -0.019 & 0.850 \\
\hline $1,25(\mathrm{OH})_{2} \mathrm{D}$ & -0.033 & 0.763 & -0.045 & 0.672 & -0.046 & 0.664 & -0.190 & 0.066 & -0.166 & 0.113 & -0.208 & 0.050 \\
\hline VFA & 0.173 & 0.097 & - & - & 0.035 & 0.770 & 0.256 & 0.021 & - & - & 0.224 & 0.056 \\
\hline Adiponectin & - & - & -0.270 & 0.008 & -0.251 & 0.039 & - & - & -0.174 & 0.123 & -0.098 & 0.403 \\
\hline$R^{2}(p)$ & 0.171 & $.020)$ & 0.211 & .004) & 0.212 & $0.007)$ & 0.278 & $0.001)$ & 0.253 & $0.001)$ & 0.284 & $0.001)$ \\
\hline
\end{tabular}

$\beta$, standardized coefficient by multiple regression analysis. $R^{2}$, coefficient of determination. Abbreviations are as in Table 1.

Model 1: adjusted for age, eGFR, serum calcium, serum phosphate, intact PTH, 1,25(OH) 2 D, and VFA.

Model 2: adjusted for age, eGFR, serum calcium, serum phosphate, intact PTH, 1,25(OH $)_{2} \mathrm{D}$, and adiponectin.

Model 3: adjusted for age, eGFR, serum calcium, serum phosphate, intact PTH, $1,25(\mathrm{OH})_{2} \mathrm{D}$, VFA, and adiponectin.

that an inverse relationship between adiponectin and FGF23 contributes to the endocrine loop between bone and adipose tissue in men. Larger studies on more participants are needed to confirm the clinical relationship between FGF23 and adiponectin. Intervention studies should also investigate whether adiponectin levels altered by dietary or pharmacological therapy directly affect serum FGF23 values.

\section{Acknowledgements}

The authors are grateful to Ms. Chiaki Murakami at the Department of Metabolism, Endocrinology, and Molecular Medicine, Osaka City University Graduate School of Medicine for technical assistance.

\section{Disclosure}

\section{Funding}

This study was supported in part by research grants from Novartis Pharma Research Grants 2017 (to TM) and Takeda Pharmaceutical Co., Ltd. (to TM) (Grant No. TKDS20180423012), Taisho Toyama Pharmaceutical Co. (to SF), Takeda Pharmaceuticals (to SF), Mitsubishi Tanabe Pharma Co. (to SF), Chugai Pharmaceutical Co. (to SF), and Astellas Pharma (to SF) (funder Id: http:// dx.doi.org/10.13039/501100004948), Osaka City University Strategic Research Grant 2014, 2015, and 2016 for top priority research (to SF), and a JSPS KAKENHI grant (number 18K11132) (to SF).

\section{Conflict of Interest}

The authors declare no competing interests related to this study. None of the funders were involved in the study design, collection, analysis, and interpretation of data, drafting the report, or the decision to submit the article for publication.

\section{References}

1. Wagner CA, Imenez Silva PH, Rubio-Aliaga I (2017) And the fat lady sings about phosphate and calcium. Kidney Int 91: 270-272.

2. Dhingra R, Sullivan LM, Fox CS, Wang TJ, D'Agostino RB Sr, et al. (2007) Relations of serum phosphorus and calcium levels to the incidence of cardiovascular disease in the community. Arch Intern Med 167: 879-885.

3. Haglin L (2001) Hypophosphataemia: cause of the dis- turbed metabolism in the metabolic syndrome. Med Hypotheses 56: 657-663.

4. Bermeo S, Gunaratnam K, Duque G (2014) Fat and bone interactions. Curr Osteoporos Rep 12: 235-242.

5. Gimble JM, Nuttall ME (2012) The relationship between adipose tissue and bone metabolism. Clin Biochem 45: 874-879.

6. Fukumoto S, Martin TJ (2009) Bone as an endocrine 
organ. Trends Endocrinol Metab 20: 230-236.

7. Kawata T, Imanishi Y, Kobayashi K, Miki T, Arnold A, et al. (2007) Parathyroid hormone regulates fibroblast growth factor-23 in a mouse model of primary hyperparathyroidism. J Am Soc Nephrol 18: 2683-2688.

8. Nakatani S, Nakatani A, Tsugawa N, Yamada S, Mori K, et al. (2015) Fibroblast growth factor-23 and vitamin D metabolism in subjects with eGFR $\geq 60 \mathrm{~mL} / \mathrm{min} / 1.73 \mathrm{~m}^{2}$. Nephron 130: 119-126.

9. Gao S, Xu J, Zhang S, Jin J (2019) Meta-analysis of the association between fibroblast growth factor 23 and mortality and cardiovascular events in hemodialysis patients. Blood Purif 47(Suppl 1): 24-30.

10. Kendrick J, Cheung AK, Kaufman JS, Greene T, Roberts WL, et al. (2011) FGF-23 associates with death, cardiovascular events, and initiation of chronic dialysis. $\mathrm{J} \mathrm{Am}$ Soc Nephrol 22: 1913-1922.

11. Marthi A, Donovan K, Haynes R, Wheeler DC, Baigent C, et al. (2018) Fibroblast growth factor-23 and risks of cardiovascular and noncardiovascular diseases: a metaanalysis. J Am Soc Nephrol 29: 2015-2027.

12. Haring R, Enserro D, Xanthakis V, Mitchell GF, Benjamin EJ, et al. (2016) Plasma fibroblast growth factor 23: clinical correlates and association with cardiovascular disease and mortality in the framingham heart study. $J \mathrm{Am}$ Heart Assoc 5: e003486.

13. Xiao Y, Luo X, Huang W, Zhang J, Peng C (2014) Fibroblast growth factor 23 and risk of all-cause mortality and cardiovascular events: a meta-analysis of prospective cohort studies. Int J Cardiol 174: 824-828.

14. Billington EO, Gamble GD, Bristow S, Reid IR (2017) Serum phosphate is related to adiposity in healthy adults. Eur J Clin Invest 47: 486-493.

15. di Giuseppe R, Kuhn T, Hirche F, Buijsse B, Dierkes J, et al. (2015) Potential predictors of plasma fibroblast growth factor 23 concentrations: cross-sectional analysis in the EPIC-Germany study. PLoS One 10: e 0133580.

16. Fernandez-Real JM, Puig J, Serrano M, Sabater M, Rubio A, et al. (2013) Iron and obesity status-associated insulin resistance influence circulating fibroblast-growth factor-23 concentrations. PLoS One 8: e58961.

17. Gutierrez OM, Wolf M, Taylor EN (2011) Fibroblast growth factor 23, cardiovascular disease risk factors, and phosphorus intake in the health professionals follow-up study. Clin J Am Soc Nephrol 6: 2871-2878.

18. Hu X, Ma X, Luo Y, Xu Y, Xiong Q, et al. (2018) Associations of serum fibroblast growth factor 23 levels with obesity and visceral fat accumulation. Clin Nutr 37: 223 228.

19. Mirza MA, Alsio J, Hammarstedt A, Erben RG, Michaelsson K, et al. (2011) Circulating fibroblast growth factor-23 is associated with fat mass and dyslipidemia in two independent cohorts of elderly individuals. Arterioscler Thromb Vasc Biol 31: 219-227.

20. Xu Y, Ma X, Pan X, He X, Xiao Y, et al. (2018) Correlations between serum concentration of three bone-derived factors and obesity and visceral fat accumulation in a cohort of middle aged men and women. Cardiovasc Diabetol 17: 143 .

21. Zaheer S, de Boer IH, Allison M, Brown JM, Psaty BM, et al. (2017) Fibroblast growth factor 23, mineral metabolism, and adiposity in normal kidney function. $J$ Clin Endocrinol Metab 102: 1387-1395.

22. Ouchi N (2016) Adipocytokines in cardiovascular and metabolic diseases. J Atheroscler Thromb 23: 645-654.

23. Kadowaki T, Yamauchi T, Kubota N, Hara K, Ueki K, et al. (2006) Adiponectin and adiponectin receptors in insulin resistance, diabetes, and the metabolic syndrome. $J$ Clin Invest 116: 1784-1792.

24. Lubkowska A, Dobek A, Mieszkowski J, Garczynski W, Chlubek D (2014) Adiponectin as a biomarker of osteoporosis in postmenopausal women: controversies. Dis Markers 2014: 975178.

25. Kanazawa I (2012) Adiponectin in metabolic bone disease. Curr Med Chem 19: 5481-5492.

26. Rutkowski JM, Pastor J, Sun K, Park SK, Bobulescu IA, et al. (2017) Adiponectin alters renal calcium and phosphate excretion through regulation of klotho expression. Kidney Int 91: 324-337.

27. Komaba H, Fukagawa M (2009) FGF23: a key player in mineral and bone disorder in CKD. Nefrologia 29: 392396.

28. Yoda K, Imanishi Y, Yoda M, Mishima T, Ichii M, et al. (2012) Impaired response of FGF-23 to oral phosphate in patients with type 2 diabetes: a possible mechanism of atherosclerosis. J Clin Endocrinol Metab 97: E2036E2043.

29. Hayashi M, Morioka T, Hatamori M, Kakutani Y, Yamazaki Y, et al. (2019) Plasma omentin levels are associated with vascular endothelial function in patients with type 2 diabetes at elevated cardiovascular risk. Diabetes Res Clin Pract 148: 160-168.

30. Zoccali C, Mallamaci F, Tripepi G, Benedetto FA, Cutrupi $\mathrm{S}$, et al. (2002) Adiponectin, metabolic risk factors, and cardiovascular events among patients with end-stage renal disease. J Am Soc Nephrol 13: 134-141.

31. American Diabetes Association (2014) Standards of medical care in diabetes-2014. Diabetes Care 37 Suppl 1: S14-S80.

32. Committee of the Japan Diabetes Society on the Diagnostic Criteria of Diabetes Mellitus, Seino Y, Nanjo K, Tajima N, Kadowaki T, et al. (2010) Report of the committee on the classification and diagnostic criteria of diabetes mellitus. J Diabetes Investig 1: 212-228.

33. Kurajoh M, Fukumoto S, Emoto M, Murase T, Nakamura $\mathrm{T}$, et al. (2020) Independent association of plasma xanthine oxidoreductase activity with serum uric acid level based on stable isotope-labeled xanthine and liquid chromatography/triple quadrupole mass spectrometry: MedCity 21 health examination registry. Clin Chem Lab Med 58: 780-786.

34. Kurajoh M, Fukumoto S, Murase T, Nakamura T, Ishihara $\mathrm{T}$, et al. (2019) Insulin resistance associated with plasma xanthine oxidoreductase activity independent of visceral 
adiposity and adiponectin level: MedCity 21 health examination registry. Int J Endocrinol 2019: 1762161.

35. Matsuo S, Imai E, Horio M, Yasuda Y, Tomita K, et al. (2009) Revised equations for estimated GFR from serum creatinine in Japan. Am J Kidney Dis 53: 982-992.

36. Nishimura M, Morioka $T$, Hayashi M, Kakutani $Y$, Yamazaki Y, et al. (2019) Plasma omentin levels are inversely associated with atherosclerosis in type 2 diabetes patients with increased plasma adiponectin levels: a crosssectional study. Cardiovasc Diabetol 18: 167.

37. Imanishi $Y$, Inaba $M$, Nakatsuka $K$, Nagasue $K$, Okuno $S$, et al. (2004) FGF-23 in patients with end-stage renal disease on hemodialysis. Kidney Int 65: 1943-1946.

38. Streicher C, Zeitz U, Andrukhova O, Rupprecht A, Pohl E, et al. (2012) Long-term Fgf23 deficiency does not influence aging, glucose homeostasis, or fat metabolism in mice with a nonfunctioning vitamin D receptor. Endocrinology 153: 1795-1805.

39. Billington EO, Murphy R, Gamble GD, Callon K, Davies $\mathrm{N}$, et al. (2019) Fibroblast growth factor 23 levels decline following sleeve gastrectomy. Clin Endocrinol (Oxf) 91: 87-93.

40. Burnett SM, Gunawardene SC, Bringhurst FR, Juppner H, Lee H, et al. (2006) Regulation of C-terminal and intact FGF-23 by dietary phosphate in men and women. $J$ Bone Miner Res 21: 1187-1196.

41. Ferrari SL, Bonjour JP, Rizzoli R (2005) Fibroblast growth factor-23 relationship to dietary phosphate and renal phosphate handling in healthy young men. $J$ Clin Endocrinol Metab 90: 1519-1524.

42. Chang AR, Lazo M, Appel LJ, Gutierrez OM, Grams ME (2014) High dietary phosphorus intake is associated with all-cause mortality: results from NHANES III. Am J Clin Nutr 99: 320-327.

43. Agbaht K, Gurlek A, Karakaya J, Bayraktar M (2009) Circulating adiponectin represents a biomarker of the associa- tion between adiposity and bone mineral density. Endocrine 35: 371-379.

44. Jurimae J, Jurimae T (2007) Adiponectin is a predictor of bone mineral density in middle-aged premenopausal women. Osteoporos Int 18: 1253-1259.

45. Kanazawa I, Yamaguchi T, Yamamoto M, Yamauchi M, Yano S, et al. (2009) Relationships between serum adiponectin levels versus bone mineral density, bone metabolic markers, and vertebral fractures in type 2 diabetes mellitus. Eur J Endocrinol 160: 265-273.

46. Lenchik L, Register TC, Hsu FC, Lohman K, Nicklas BJ, et al. (2003) Adiponectin as a novel determinant of bone mineral density and visceral fat. Bone 33: 646-651.

47. Richards JB, Valdes AM, Burling K, Perks UC, Spector TD (2007) Serum adiponectin and bone mineral density in women. J Clin Endocrinol Metab 92: 1517-1523.

48. Berner HS, Lyngstadaas SP, Spahr A, Monjo M, Thommesen L, et al. (2004) Adiponectin and its receptors are expressed in bone-forming cells. Bone 35: 842-849.

49. Shinoda Y, Yamaguchi M, Ogata N, Akune T, Kubota N, et al. (2006) Regulation of bone formation by adiponectin through autocrine/paracrine and endocrine pathways. $J$ Cell Biochem 99: 196-208.

50. Feng JQ, Ye L, Schiavi S (2009) Do osteocytes contribute to phosphate homeostasis? Curr Opin Nephrol Hypertens 18: 285-291.

51. Oh KW, Lee WY, Rhee EJ, Baek KH, Yoon KH, et al. (2005) The relationship between serum resistin, leptin, adiponectin, ghrelin levels and bone mineral density in middle-aged men. Clin Endocrinol (Oxf) 63: 131-138.

52. Tsuji K, Maeda T, Kawane T, Matsunuma A, Horiuchi N (2010) Leptin stimulates fibroblast growth factor 23 expression in bone and suppresses renal 1alpha,25dihydroxyvitamin D3 synthesis in leptin-deficient mice. $J$ Bone Miner Res 25: 1711-1723. 